\title{
Solute transport through porous media using asymptotic dispersivity
}

\author{
P K SHARMA* and TEODROSE ATNAFU ABGAZE \\ Department of Civil Engineering, Indian Institute of Technology Roorkee, \\ Roorkee 247 667, India \\ e-mail: drpksharma07@gmail.com; asgtedi@yahoo.com
}

MS received 9 August 2014; revised 13 January 2015; accepted 21 January 2015

\begin{abstract}
In this paper, multiprocess non-equilibrium transport equation has been used, which accounts for both physical and chemical non-equilibrium for reactive transport through porous media. An asymptotic distance dependent dispersivity is used to embrace the concept of scale-dependent dispersion for solute transport in heterogeneous porous media. Semi-analytical solution has been derived of the governing equations with an asymptotic distance dependent dispersivity by using Laplace transform technique and the power series method. For application of analytical model, we simulated observed experimental breakthrough curves from $1500 \mathrm{~cm}$ long soil column experiments conducted in the laboratory. The simulation results of breakthrough curves were found to deviate from the observed breakthrough curves for both mobile-immobile and multiprocess non-equilibrium transport with constant dispersion models. However, multiprocess non-equilibrium with an asymptotic dispersion model gives better fit of experimental breakthrough curves through long soil column and hence it is more useful for describing anomalous solute transport through heterogeneous porous media. The present model is simpler than the stochastic numerical method.
\end{abstract}

Keywords. Analytical solution; asymptotic dispersivity; heterogeneous soil column; breakthrough curves.

\section{Introduction}

Solute transport in soil and groundwater systems is affected by various factors such as physical, chemical, and microbiological processes. The transport of chemical is affected by a large number of processes and porous media properties including convective transport with flowing water, molecular diffusion, hydrodynamic dispersion, equilibrium or non-equilibrium exchange with the solid phase if reactive solutes are involved, and possibly production and decay processes.

${ }^{*}$ For correspondence 
Several mathematical models have been developed to represent the process of transport of reactive contaminants through porous media (Valocchi 1985; Goltz \& Roberts 1986; Brusseau et al 1989).

Over the last few decades, it is known that distance-dependent dispersion has long been a focus of experimental and theoretical research on solute transport through porous media. The essence of distance-dependent dispersion is that the dispersion coefficients obtained from the analysis of the breakthrough curves by the convection-dispersion equation (CDE) are not constant but increase with travel distance. The greater the travel distance in a tracer test used to measure dispersivity, the larger the dispersivity value that is calculated by Molz et al (1983). This type of phenomenon occurred in the field-scale transport and in laboratory-scale transport (Pickens \& Grisak 1981a; Gelhar et al 1992; Khan \& Jury 1990; Yates 1990, 1992; Huang et al 1995; Schulze-Makuch 2005; Vanderborght \& Vereecken 2007). The dispersivities obtained from laboratory observation are usually on the order of a few millimeters to tens of millimeters, while those from the field can be several orders of magnitude higher (Pickens \& Grisak 1981a; Gelhar et al 1992; Vanderborght \& Vereecken 2007). A general consensus at present is that the scale-dependent dispersion is used to represent the heterogeneous nature of porous media at different scales (Gelhar et al 1992; Huang et al 2006). Hence, CDE with a constant dispersivity cannot satisfactorily describe solute transport at various scales in soil and groundwater systems (Pickens \& Grisak 1981b; Gao et al 2010). Most of the models were initially based on the classical one-dimensional CDE as studied by Lapidus \& Amundson (1952) and van Genuchten \& Wierenga (1977). Later they considered one-dimensional and multi-dimensional model for increasing complicated process and soil properties. This was done by extending the models to include different types of sorption sites, a variety of expressions characterizing physical and chemical non-equilibrium, and alternative geometries of immobile (stagnant) liquid phases in the medium (De Smedt \& Wierenga 1979; Valocchi 1985; Brusseau et al 1989). Most of the studies focused on the formulation of appropriate mass balance (partial differential) equations governing solute transport through porous media. Huang et al (1996) derived analytical solutions for one-dimensional transport with linear asymptotic scale-dependent dispersion. They assumed that the dispersivity increases linearly with distance until some travel distance after which the dispersivity reached an asymptotic value. Conservative solute transport in porous media is typically modeled using the generalized advection and dispersion equation (ADE) (Bear 1972). The solution of ADE's in most groundwater transport applications requires numerical methods, except the limited cases where analytical solutions exist (van Genuchten \& Alves 1982). Analytical solutions have an important role to play because they offer fundamental insight into governing physical processes, provide useful tools for validating numerical solutions, and are sometimes more computationally efficient. Most previously published analytical solutions to advective-dispersive transport problems are predicated on the assumption of a homogeneous porous medium (van Genuchten \& Alves 1982). In reality, subsurface porous media is seldom homogeneous and significant spatial variability of transport properties can be expected (Gelhar 1993). However, the use of CDE has been frequently questioned by many investigators in recent years, as it cannot adequately account for anomalous transport in heterogeneous porous media, and alternative models have been proposed. The mobile-immobile model (MIM) presented by van Genuchten \& Wierenga (1976) is a practical and physically based approach to describe anomalous solute transport behavior. A model that accounts for multiple sources of non-equilibrium would be required to accurately represent these systems. Such model was developed by Brusseau et al (1989). Additional terms in the advection-dispersive equation making it more generally applicable in the modeling of dead end pores in the medium. The liquid filled dead end pores were called immobile water. Transfer of solute mass takes place 
between the mobile and immobile liquid zones. Brusseau et al (1989) developed multiprocess non-equilibrium (MPNE) model that includes physical and chemical non-equilibrium in reactive solute movement through the porous media. The physical non-equilibrium in transport is caused due to preferential and stagnant flow. The chemical non-equilibrium is caused due to the simultaneous presence of instantaneous and rate limited sorption of the solute in the solid and liquid forms. Brusseau et al (1992) used advective-dispersive reactive transport model that explicitly accounts for multiple sources of non-equilibrium and transformation reactions during steady state flow in porous media. Logan (1996) derived analytical solution for one-dimensional governing equation for the transport of solute tracer in heterogeneous porous medium considering rate-limited sorption with a linear equilibrium isotherm, and decay. Chen et al (2003) derived an analytical solution for two-dimensional non-axis symmetric solute transports in a radially convergent flow field. Laplace transformed power series (LTPS) technique is used to solve the two-dimensional ADEs in cylindrical coordinates. The analytical power series solution was compared to the numerical solution of Chen et al (1999). The two-dimensional power series solution is appropriate for hydro-geologic circumstances. Furman \& Neuman (2003) proposed Laplace-transform analytic element method (LT-AEM) for the solution of transient flow problems in porous media. Analytic element method (AEM) is used to solve the resultant timeindependent modified Helmholtz equation, and the solution is inverted numerically back into the time domain. Huang et al (2006) developed semi-analytical inverse technique and program of fractional advection-dispersion equation (FADE) for parameter estimation of the FADE. They analyzed laboratory experimental data of conservative solute transport in $12.5 \mathrm{~m}$ long homogeneous and heterogeneous soil columns to test the non-Fickian dispersion theory of FADE. The scale effect of the dispersion coefficient in the heterogeneous soil was much more significant comparing to that in the homogeneous soil. Chen et al (2008) used hyperbolic asymptotic distance dependent dispersivity, which initially increases with travel distance and eventually reaches an asymptotic value at long travel distance. Moreover, breakthrough curves obtained from the hyperbolic asymptotic dispersivity model are compared with those obtained from the constant dispersivity model to scrutinize the relationship of the transport parameters derived by Mishra \& Parker (1990).

Gao et al (2009a, b) compared five different models for evaluating solute transport in a $1250-\mathrm{cm}$ long saturated and heterogeneous soil column. These models are CDE, MIM, convective log normal transfer function model (CLT), the spatial FADE and the continuous time random walk model and were also used to fit the BTC at different distances simultaneously. This study indicated that CDE, CLT and FADE were unable to describe the anomalous breakthrough curves adequately and their parameters changed with transport distance. Guerrero \& Skaggs (2010) derived analytical solution for the linear, one-dimensional advection dispersion equation with distance dependent coefficients. It is shown that an analytical expression for the integration factor exists for several transport formulations of practical importance in groundwater transport modeling. Gao et al (2010) proposed MIM to describe reactive solute transport with scale dependent dispersion in heterogeneous porous media. The proposed model incorporates distance-dependent dispersion with linear and exponential dispersivity. They concluded that MIM with scale-dependent dispersion assuming the dispersivity to be an exponential function of distance is a simple and practical approach to describe solute in heterogeneous porous media. Gao et al (2012) combined the MIM with an asymptotic dispersivity function of travel distance to embrace the concept of scale-dependent dispersion during solute transport in finite heterogeneous porous media.

From the above literature review, it is seen that analytical solution for MPNE transport equation is not developed using asymptotic dispersivity function. Therefore, in this study 
an attempt has been made to derive semi-analytical solution of MPNE transport equations using power series method with asymptotic distance-dependent dispersion model for constant concentration type boundary condition. Analytical model has been used to simulate the experimental breakthrough curves of both reactive and non-reactive solutes through long soil column experiments.

\section{Governing equations}

The transport of reactive solute is influenced by transport related and sorption related nonequilibrium processes (Brusseau et al 1989). MPNE model is formulated for the cases where non-equilibrium is caused by a combination of transport and sorption related processes. The transport related non-equilibrium is also known as physical non-equilibrium, results from the existence of regions within the porous medium in which there is minimum advective flow. Sorption related non-equilibrium occurs due to both from chemical non-equilibrium and intrasorbent diffusion. Chemical non-equilibrium results from rate-limited interactions between the solute and specific sorption sites of both the sorbent. Non-equilibrium resulting from intrasorbent diffusion involves the diffusive mass transfer of sorbate within the sorbent matrix. The sorption dynamics of model consists of two serially arranged bicontinuum coupled in parallel. It discretizes the porous medium into four sorption domains, where instantaneous sorption occurs in the first domain and rate-limited sorption occurs in the other three domains. Based on this conceptual model, Brusseau et al (1992) developed general transport equations that account for both the physical and chemical non-equilibrium for reactive solute transport through porous media. The advective-dispersive-reactive transport equation for transport of solute through the advective region of a porous media can be written as

$$
\begin{aligned}
\left(\theta_{a}+f \rho F_{a} K_{a}\right) \frac{\partial C_{a}}{\partial t}+f \rho \frac{\partial S_{a 2}}{\partial t}= & \frac{\partial}{\partial x}\left[\theta_{a} D(x) \frac{\partial C_{a}}{\partial x}\right]-q \frac{\partial C_{a}}{\partial x}-w\left(C_{a}-C_{n}\right)- \\
& \left(\mu_{a} \theta_{a}+\mu_{a 1} f \rho F_{a} K_{a}\right) C_{a}-\mu_{a 2} f \rho S_{a 2} .
\end{aligned}
$$

The mass balance for the non-advective region can be described by the following equation:

$$
\begin{gathered}
\left(\theta_{n}+(1-f) \rho F_{n} K_{n}\right) \frac{\partial C_{n}}{\partial t}+(1-f) \rho \frac{\partial S_{n 2}}{\partial t}=w\left(C_{a}-C_{n}\right)-\left(\mu_{n} \theta_{n}+\mu_{n 1}(1-f) \rho F_{n} K_{n}\right) C_{n}- \\
\mu_{n 2}(1-f) \rho S_{n 2},
\end{gathered}
$$

where $x$ is the spatial coordinate $(\mathrm{L}), \theta_{a}$ is the fractional volumetric water content of the advective region $\left(\mathrm{L}^{3} / \mathrm{L}^{3}\right), \theta_{n}$ is the fractional volumetric water content of the non-advective region $\left(\mathrm{L}^{3} / \mathrm{L}^{3}\right), f$ is the mass fraction of sorbent constituting the advective region, $\rho$ is the bulk density of the porous medium $\left(\mathrm{ML}^{-3}\right), F_{a}$ and $F_{n}$ are the mass fraction of sorbent for which sorption is essentially instantaneous, $K_{a}$ and $K_{n}$ are the equilibrium sorption coefficient for advective and non-advective regions $\left(\mathrm{L}^{3} \mathrm{M}^{-1}\right), C_{a}$ is the concentration of solute in the solution phase for advective region $\left(\mathrm{ML}^{-3}\right), C_{n}$ is the concentration of solute in solution phase for non-advective region ( $\mathrm{ML}^{-3}$ ), $t$ is the time (T), $S_{a 2}$ and $S_{n 2}$ are the rate-limited sorbed-phase concentration in advective and non-advective regions, respectively $\left(\mathrm{MM}^{-1}\right), q$ is the specific discharge (LT ${ }^{-1}$ ), $w$ is the first-order coefficient for mass transfer between the advective and non-advective regions $\left(\mathrm{T}^{-1}\right), \mu_{a}$ and $\mu_{n}$ are the first-order transformation coefficient for the solution phase in advective and non-advective regions, $\mu_{a 1}$ and $\mu_{n 1}$ are the transformation coefficients $\left(\mathrm{T}^{-1}\right)$ for the instantaneous sorbed-phase of advective and non-advective regions, respectively, and $\mu_{a 2}$ and $\mu_{n 2}$ are the transformation coefficients for rate-limited sorbed-phase domains of advective and non-advective regions, respectively $\left(\mathrm{T}^{-1}\right)$. 
Dynamics of sorption and transformation for the rate-limited domains are described by

$$
\begin{aligned}
& \frac{\partial S_{a 2}}{\partial t}=k_{a 2}\left[\left(1-F_{a}\right) K_{a} C_{a}-S_{a 2}\right]-\mu_{a 2} S_{a 2} \\
& \frac{\partial S_{n 2}}{\partial t}=k_{n 2}\left[\left(1-F_{n}\right) K_{n} C_{n}-S_{n 2}\right]-\mu_{n 2} S_{n 2},
\end{aligned}
$$

where $k_{\mathrm{a} 2}$ and $k_{\mathrm{n} 2}$ are the first-order reverse sorption rate coefficients $\left(\mathrm{T}^{-1}\right)$ for the advective and non-advective regions, respectively.

It is generally accepted that the solute transport can be well described by the convection dispersion equation for laboratory column packed with homogeneous soils (Bear 1972). However, in heterogeneous soils the measured breakthrough curves usually show early arrival and long tailing due to the inherent heterogeneity of natural soils at various scales (Berkowitz et al 2006). Such phenomena can be found during solute transport in repacked soil column. It is also recognized that the estimated dispersivity is not constant but varies with spatial scales (Gelhar et al 1992). Even if the field is macroscopically homogeneous, the dispersion coefficient is not a constant from the beginning, but only after the tracer plume has been transported over a large enough domains, containing several correlation lengths (Dentz et al 2000). Therefore, it is difficult for convection dispersion equation with a single set of parameters to predict breakthrough curves at different distances. Hence, asymptotic dispersivity function of travel distance is used to embrace the concept of scale-dependent dispersion during solute transport in heterogeneous media.

The hydrodynamic dispersion coefficient $D(x)$ is a function of distance in the porous media $\left(\mathrm{L}^{2} \mathrm{~T}^{-1}\right)$. It is commonly expressed as follows without consideration of molecular diffusion (Bear 1972):

$$
D(x)=\alpha(x) V_{a},
$$

where $\alpha(x)$ is the dispersivity (L) and $V_{a}=q / \theta_{a}$ is the pore water velocity in advective region. It is observed that the dispersivity generally increases with transport distance in the subsurface groundwater systems. However, an asymptotic dispersivity is considered to account for the heterogeneity of porous media. For this case, the dispersivity is assumed to initially increase with travel distance and approach an asymptotic value eventually.

Asymptotic distance-dependent dispersion coefficient is given by (Pickens \& Grisak 1981b):

$$
D(x)=a\left(1-\frac{b}{x+b}\right) V_{a},
$$

where $a$ is an asymptotic dispersivity value $(L)$ and $b$ is a characteristic distance $(L)$ which determine the travel distance for the dispersivity to reach half of the asymptotic value. Asymptotic dispersivity is the ratio of asymptotic dispersion coefficient to the pore water velocity. Figure 1 shows the spatial variation of the asymptotic dispersion function equal to the ratio between distance-dependent dispersion coefficient and asymptotic dispersivity for different values of $b$. The values of $\mathrm{b}$ depend on the extent of the pre-asymptotic zone. For a smaller value of $b$ the dispersivity will approach the asymptotic value faster. The value $b$ equal to zero indicates the constant dispersion.

\section{Initial and boundary conditions}

In the present study, we consider solute transport in a finite system with a length of $L$. The initial solute concentrations are assumed to be uniform with $C_{i}$ for the entire domain. It is assumed 


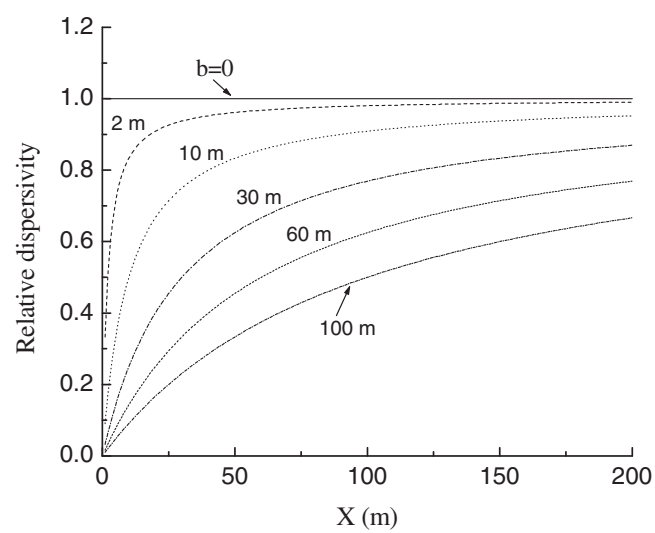

Figure 1. Variation of relative dispersivity with different values of characteristic distance (b).

that the initial rate-limited sorbed-phase concentrations $S_{i}$ (at time $\mathrm{t}=0$ ) are same in both the mobile and immobile regions of the porous medium. The zero gradient boundary condition is imposed at the exit, which can satisfy the mass balance requirement and ensure the continuity in concentration at $x=L$. The initial and the exit boundary conditions can be given as

$$
\begin{gathered}
C_{a}(x, 0)=C_{n}(x, 0)=C_{i}, \quad 0 \leq x \leq L \\
\frac{\partial C_{a}(L, t)}{\partial x}=0, \quad 0 \leq t \leq \infty .
\end{gathered}
$$

Constant concentration condition at inlet can be given as

$$
C_{a}(0, t)=C_{0}, \quad 0 \leq t \leq \infty,
$$

where $C_{0}$ is the constant source concentration $\left(\mathrm{ML}^{-3}\right)$. The value of dispersion coefficient, $D(x)$ is equal to zero at $x=0$.

\section{Semi-analytical solution}

Taking Laplace transform of Eq. (3) with respect to time $t$

$$
P \overline{S_{a 2}}-S_{i}=k_{a 2}\left[\left(1-F_{a}\right) K_{a} \overline{C_{a}}-\overline{S_{a 2}}\right]-\mu_{a 2} \overline{S_{a 2}} \text {. }
$$

After simplifying,

$$
\overline{S_{a 2}}=\frac{\left[k_{a 2}\left(1-F_{a}\right) K_{a}\right] \overline{C_{a}}+S_{i}}{P+k_{a 2}+\mu_{a 2}} .
$$

Similarly, Laplace transform of Eq. (4) can be written in simplified form as

$$
\overline{S_{n 2}}=\frac{\left[k_{n 2}\left(1-F_{n}\right) K_{n}\right] \overline{C_{n}}+S_{i}}{P+k_{n 2}+\mu_{n 2}} .
$$

Laplace transform of Eq. (2) can be written as

$$
\begin{aligned}
R_{n} P \overline{C_{n}} & -R_{n} C_{i}+(1-f) \rho P \overline{S_{n 2}}-(1-f) \rho S_{i} w\left(\overline{C_{a}}-\overline{C_{n}}\right) \\
& -\left[\mu_{n} \theta_{n}+\mu_{n 1}(1-f) \rho F_{n} K_{n}\right] \overline{C_{n}}-\mu_{n 2}(1-f) \rho \overline{S_{n 2}} .
\end{aligned}
$$


Substituting the value of $\overline{S_{n 2}}$ from Eq. (10) into Eq. (11) and after simplifying following expression can be obtained as

$$
\overline{C_{n}}=\frac{w\left(P+k_{n 2}+\mu_{n 2}\right) \overline{C_{a}}+R_{n}\left(P+k_{n 2}+\mu_{n 2}\right) C_{i}+(1-f) \rho k_{n 2} S_{i}}{R_{n} P^{2}+A_{1} P+A_{2}},
$$

where coefficients:

$$
\begin{gathered}
A_{1}=R_{n}\left(k_{n 2}+\mu_{n 2}\right)+w+\mu_{n} \theta_{n}+\mu_{n l}(1-f) \rho F_{n} K_{n}+(1-f)\left(1-F_{n}\right) \rho K_{n} k_{n 2} \\
A_{2}=\left(w+\mu_{n} \theta_{n}+\mu_{n l}(1-f) \rho F_{n} K_{n}\right)\left(\left(k_{n 2}+\mu_{n 2}\right)+(1-f)\left(1-F_{n}\right) \rho K_{n} k_{n 2}\right. \\
R_{a}=\theta_{a}+f \rho F_{a} K_{a} \\
R_{n}=\theta_{n}+(1-f) \rho F_{n} K_{n} .
\end{gathered}
$$

Taking Laplace-transform of Eq. (1) and after simplifying:

$$
\begin{array}{r}
\theta_{a} D(x) \frac{d^{2} \overline{C_{a}}}{d x^{2}}+\left(\theta_{a} \frac{\partial D(x)}{\partial x}-q\right) \frac{d \overline{C_{a}}}{d x}-\overline{C_{a}}\left(w+\mu_{a} \theta_{a}+\mu_{a l} f \rho F_{a} K_{a}+R_{a} P\right)+w \overline{C_{n}}- \\
\overline{S_{a 2}}\left(\mu_{a 2} f \rho+f \rho P\right)+R_{a} C_{i}+f \rho S_{i}=0,
\end{array}
$$

where $R_{a}=\theta_{a}+f \rho F_{a} K_{a}$, and after substituting the value of $\overline{C_{n}}$ from Eq. (11) and $\overline{S_{a 2}}$ from Eq. (9) into above Eq. (14) and simplified form can be written as

$$
D(x) \frac{d^{2} \overline{C_{a}}}{d x^{2}}+\left(\theta_{a} \frac{\partial D(x)}{\partial x}-q\right) \frac{d \overline{C_{a}}}{d x}-A_{3} \overline{C_{a}}+A_{4}=0,
$$

where

$$
\begin{aligned}
& A_{3}=w+\mu_{a} \theta_{a}+\mu_{a l} f \rho F_{a} K_{a}+R_{a} P-\frac{w^{2}\left(P+k_{n 2}+\mu_{n 2}\right)}{R_{n} P^{2}+A_{1} P+A_{2}}+\frac{f \rho\left(\mu_{a 2}+P\right) k_{a 2} K_{a}\left(1-F_{a}\right)}{\left(P+k_{a 2}+\mu_{a 2}\right)} \\
& A_{4}=C_{i}\left[R_{a}+\frac{w R_{n}\left(P+k_{n 2}+\mu_{n 2}\right)}{R_{n} P^{2}+A_{1} P+A_{2}}\right]+S_{i}\left[\frac{w(1-f) \rho k_{n 2}}{R_{n} P^{2}+A_{1} P+A_{2}}-\frac{f \rho\left(\mu_{a 2}+P\right)}{\left(P+k_{a 2}+\mu_{a 2}\right)}+f \rho\right] .
\end{aligned}
$$

Substituting the value of $D(x)=a\left(1-\frac{b}{x+b}\right) V_{a}$ into Eq. (15) and yields the following final simplified equation:

$$
\frac{(a x) V_{a}}{(x+b)} \frac{d^{2} \overline{C_{a}}}{d x^{2}}-V_{a}\left[1-\frac{(a b)}{(x+b)^{2}}\right] \frac{d \overline{C_{a}}}{d x}-\frac{A_{3}}{\theta_{a}}\left[\overline{C_{a}}-\frac{A_{4}}{A_{3}}\right]=0 .
$$

Defining, $Y=\overline{C_{a}}-\frac{A_{4}}{A_{3}}$, Eq. 17 can be changed to the following equation:

$$
\frac{(a x) V_{a}}{(x+b)} \frac{d^{2} Y}{d x^{2}}-V_{a}\left[1-\frac{(a b)}{(x+b)^{2}}\right] \frac{d Y}{d x}-\psi Y=0,
$$

where $\psi=A_{3} / \theta_{a}$. 
The solution of Eq. (18) can be described by power series method by Kreyszig (1999) and Chen et al (2008). Following the similar approach of Chen et al (2008) and Gao et al (2012), the solution of MPNE in the Laplace domain can be obtained as

$$
\overline{C_{a}}=A_{1} Y_{1}(x, p)+\mathrm{B}_{1} \mathrm{Y}_{2}(\mathrm{x}, \mathrm{p})+\frac{\mathrm{C}_{\mathrm{i}}}{\mathrm{P}}
$$

In terms of the inlet and exit boundary conditions, $\mathrm{A}_{1}$ and $\mathrm{B}_{1}$ can be easily obtained.

$$
\begin{gathered}
A_{1}=\frac{C_{0}-C_{i}}{P} \\
B_{1}=\left(\frac{C_{i}-C_{0}}{P}\right) \frac{\frac{d Y_{1}(L, P)}{d x}}{\frac{d Y_{2}(L, P)}{d x}} .
\end{gathered}
$$

With respect to the derived solutions, coefficient $a_{0}$ in $Y_{1}(\mathrm{x}, \mathrm{p})$ and $Y_{2}(\mathrm{x}, \mathrm{p})$ can be any nonzero constant value as $a_{1}, a_{2}, \ldots, a_{\mathrm{m}}$ all can be expressed as a linear function of $a_{0}$ in terms of equations (a6-a7) of Gao et al (2012). In this study, the coefficient $a_{0}$ is set to be 1 . The required terms of series solution to achieve convergence depend on the Peclet number. Chen et al (2002) demonstrated that the numbers of required terms for the power series solutions were around 50, 120, and 300 when Peclet numbers equal to 1, 10 and 100, respectively. After derived solutions in Laplace domain, we need to transform them into real time domain by using numerical Laplace inversion method. In this paper, the numerical inversion of Laplace transform is done by de Hoog algorithm (de Hoog et al 1982). The de Hoog's algorithm has been widely applied on numerous flow and transport problems and has been found to perform satisfactory on both advection and dispersion dominated cases (Moench 1991; Park \& Zhan 2003; Furman \& Neuman 2003; Gao et al 2010) (figure 2).

\section{Validation of the semi-analytical solution}

The analytical solution for MPNE transport equation is not available in the literature for asymptotic dispersivity. Hence, present semi-analytical solution of MPNE model is validated for

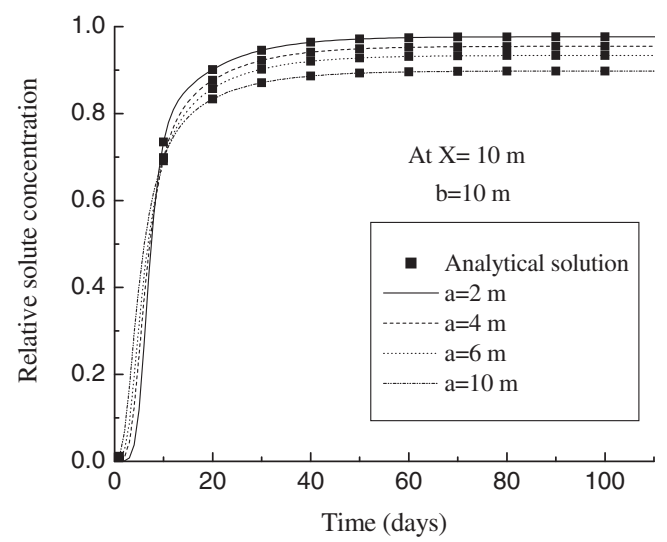

Figure 2. Comparison of the concentration profile at $10 \mathrm{~m}$ obtained from semi-analytical solution with different values of 'a' for a fixed value of 'b' equal to $10 \mathrm{~m}$. 


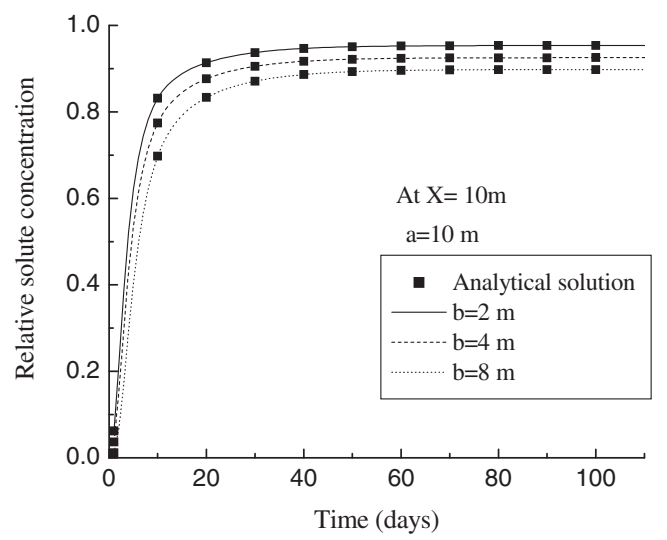

Figure 3. Comparison of the concentration profile at $10 \mathrm{~m}$ obtained from semi-analytical solution with different values of ' $b$ ' for a fixed value of ' $a$ ' equal to $10 \mathrm{~m}$.

simplified case of mobile-immobile (MIM) with asymptotic dispersivity of Gao et al (2012). In the comparison cases, adsorption of the solute is not considered and the initial concentration in the medium is taken equal to zero. The length of finite system is taken as $10 \mathrm{~m}$. The flow rate $(q)$ is $0.4 \mathrm{~m} /$ day. The water content of the medium system is taken to be $\theta=0.4$ and water content in the mobile region equal to $\theta_{a}=0.3$ and in immobile region is $\theta_{n}=0.1$. The value of mass transfer coefficient (w) is 0.01 per day. The comparison tests are conducted under various combinations of $a$ and $b$ values which are key parameters. Figure 2 shows the comparison of concentration profile obtained at $10 \mathrm{~m}$ from present semi-analytical solution and with different values of $a(a=2 \mathrm{~m}, 4 \mathrm{~m}, 6 \mathrm{~m}$ and $10 \mathrm{~m})$ and a fixed value of $b=10 \mathrm{~m}$. Similar, comparison results are shown in figure 3 with different values of $b(b=2 \mathrm{~m}, 4 \mathrm{~m}$, and $8 \mathrm{~m})$ and a fixed value of $a=10 \mathrm{~m}$. Comparison results between present solution as compared with the analytical solution gives very good match.

\section{Application of model}

\subsection{Solute transport experiments in long soil column}

For applicability of developed model, laboratory experiments were conducted on a $1500 \mathrm{~cm}$ long horizontally placed heterogeneous soil column. Sodium chloride and sodium fluoride are used as non-reactive and reactive tracers in the experiments. A horizontally placed column made-up of acrylic pipe with size of $6 \mathrm{~mm}$ thickness, having $15 \mathrm{~cm}$ diameter and $1500 \mathrm{~cm}$ long is considered. The soil column is packed with soil mixtures of natural soil, gravel, fine sand and coarse sand in equal proportion by weight. The mean particle size $\left(D_{50}\right)$ of natural soil is $0.33 \mathrm{~mm}$, gravel is $6 \mathrm{~mm}$, fine sand is $0.75 \mathrm{~mm}$ and coarse sand is $1.1 \mathrm{~mm}$. Prior to filing, the soil material was cleaned, washed and dried to ensure that the material free from organic matters. The soil column was packed with mixture of materials in layer by layer to get the uniform density throughout the column length. During packing, piezometers were installed laterally at $100 \mathrm{~cm}$ intervals in the center along the length of column. The horizontally placed soil column was fully saturated slowly with tap water for three to four days, and the steady-state water flow condition was established by using peristaltic pump. The soil column was saturated slowly from inlet 
of the soil column with deaired tap water during start of the solute transport experiment. Thus, entrapped air in the soil column was removed. A peristaltic pump is used to obtain a steady saturated flow in the soil column. Sodium chloride $(\mathrm{NaCl})$ solution with initial solute concentration of $\mathrm{C}_{0}=60 \mathrm{mg} / \mathrm{L}$ was injected into the soil column through peristaltic pump. Similarly fluoride with an initial solute concentration of $5 \mathrm{mg} / \mathrm{L}$ in solution phase was injected at the inlet of soil column through peristaltic pump.

A continuous concentration type input is maintained at the inlet of the column and solute concentration in solution phase was measured for different time intervals at a distance of 300 $\mathrm{cm}, 600 \mathrm{~cm}, 900 \mathrm{~cm}, 1200 \mathrm{~cm}$ and $1500 \mathrm{~cm}$ along the soil column. The estimated value of total volumetric water content, i.e. $\theta=\left(\theta_{a}+\theta_{n}\right)$ of the soil media within the column was measured as 0.38 , and the observed flow rate $(q)$ at the outlet of the soil column was $0.326 \mathrm{~cm} / \mathrm{min}$. The computed value of bulk density $(\rho)$ of the soil media was found to be equal to $1.74 \mathrm{~g} / \mathrm{cm}^{3}$.

\subsection{Parameter estimation and simulation of experimental data of chloride}

The MPNE model is identical to MIM model for non-reactive transport through porous media. The breakthrough curves of Chloride are observed at $300 \mathrm{~cm}, 600 \mathrm{~cm}, 1200 \mathrm{~cm}$ and $1500 \mathrm{~cm}$ down gradient in the flow direction. These data of experimental breakthrough curves are simulated using mobile-immobile model with constant dispersivity (MIMC) and mobile-immobile model with asymptotic dispersivity (MIMA). There are three parameters in MIMC model i.e., $\left(\theta_{a}, w\right.$ and $\left.D\right)$ and four parameters $\left(\theta_{a}, w, a\right.$ and $\left.b\right)$ in MIMA. However, MIMC and MIMA models have similar mobile water fraction and mass transfer coefficient values. The difference between them was that MIMC has a constant dispersion coefficient in the mobile region whereas the dispersivity in MIMA has an asymptotic distance-dependent function with parameters of $a$ and $b$. Thus, in the simulation process, the Levenberg-Marquardt nonlinear least square optimization approach was firstly used to fit the break through curve at $1500 \mathrm{~cm}$ with MIMC to determine the values of $\theta_{a}, w$ and $D$. The fixed value of $\theta_{a}$ and w, the other two parameters ( $a$ and $b$ ) in MIMA are determined by fitting it with breakthrough curve at $1500 \mathrm{~cm}$.

Firstly, the experimental breakthrough of chloride obtained at $1500 \mathrm{~cm}$ was fitted using MIMC model with constant dispersion model. Estimated value of dispersivity was found to be equal to $108.56 \mathrm{~cm}$, the value of mass transfer coefficient, $w=2.31 \mathrm{E}-05 \mathrm{~min}^{-1}$ and the value of water content in the mobile region was $\theta_{a}=0.34$. Other measured parameters were kept same as measured. Now the experimental BTC was simulated with constant and asymptotic distancedependent dispersion models. The model parameters are estimated with optimization algorithm and their values were $a=82.73 \mathrm{~cm}, b=148.21 \mathrm{~cm}$ for asymptotic distance dependent model. The estimated model parameters for constant and asymptotic distance-dependent dispersion models are listed in table 1. Figure 4a shows the fitted BTC at $1500 \mathrm{~cm}$ distance with constant (MIMC) and asymptotic distance dependent (MIMA) dispersion models. It can be seen that constant dispersion model tends to overestimate the BTC at small transport time. Also, the estimated value of coefficient of correlation $r^{2}=0.9754$ and RMSE $=0.08135$ for MIMC model, and value $r^{2}=0.9964$ and root mean square error, $\mathrm{RMSE}=0.02631$ are obtained for the MIMA model. This value indicates that the value of coefficient of correlation is higher for MIMA model as compared to MIMC model. Thus, indicating the unsuitability of MIMC to simulate the BTC as compared to MIMA model. These estimated model parameters for constant and asymptotic distance dependent are kept fixed and the experimental BTC at $300 \mathrm{~cm}, 600 \mathrm{~cm}$ and $1200 \mathrm{~cm}$ are simulated using these parameters. The simulated experimental breakthrough curves at 300 $\mathrm{cm}, 600 \mathrm{~cm}$ and $1200 \mathrm{~cm}$ are shown in figure 4b, 4c, and 4d. The estimated value of coefficient of correlation and RMSE is shown in table 2 for MIMC and MIMA dispersion models. It can be 
Table 1. Model parameters used for simulation of Chloride BTC.

\begin{tabular}{lc}
\hline Parameters & MIM model \\
\hline$q(\mathrm{~cm} / \mathrm{min})$ & 0.326 \\
$\theta_{\mathrm{a}}$ & 0.34 \\
$\theta_{\mathrm{n}}$ & 0.04 \\
$w\left(\mathrm{~min}^{-1}\right)$ & $2.31 \mathrm{E}-05$ \\
Dispersivity $(\mathrm{cm})$ & 108.56 \\
Asymptotic distance dependent coefficient & $A=82.73 \mathrm{~cm}$ \\
& $b=148.21 \mathrm{~cm}$
\end{tabular}

seen from figure 4(b-d) that a higher arrival time is observed with constant dispersion model as compared to MIMA model. A much better fit was obtained with asymptotic distance dependent model as compared to constant dispersion model.
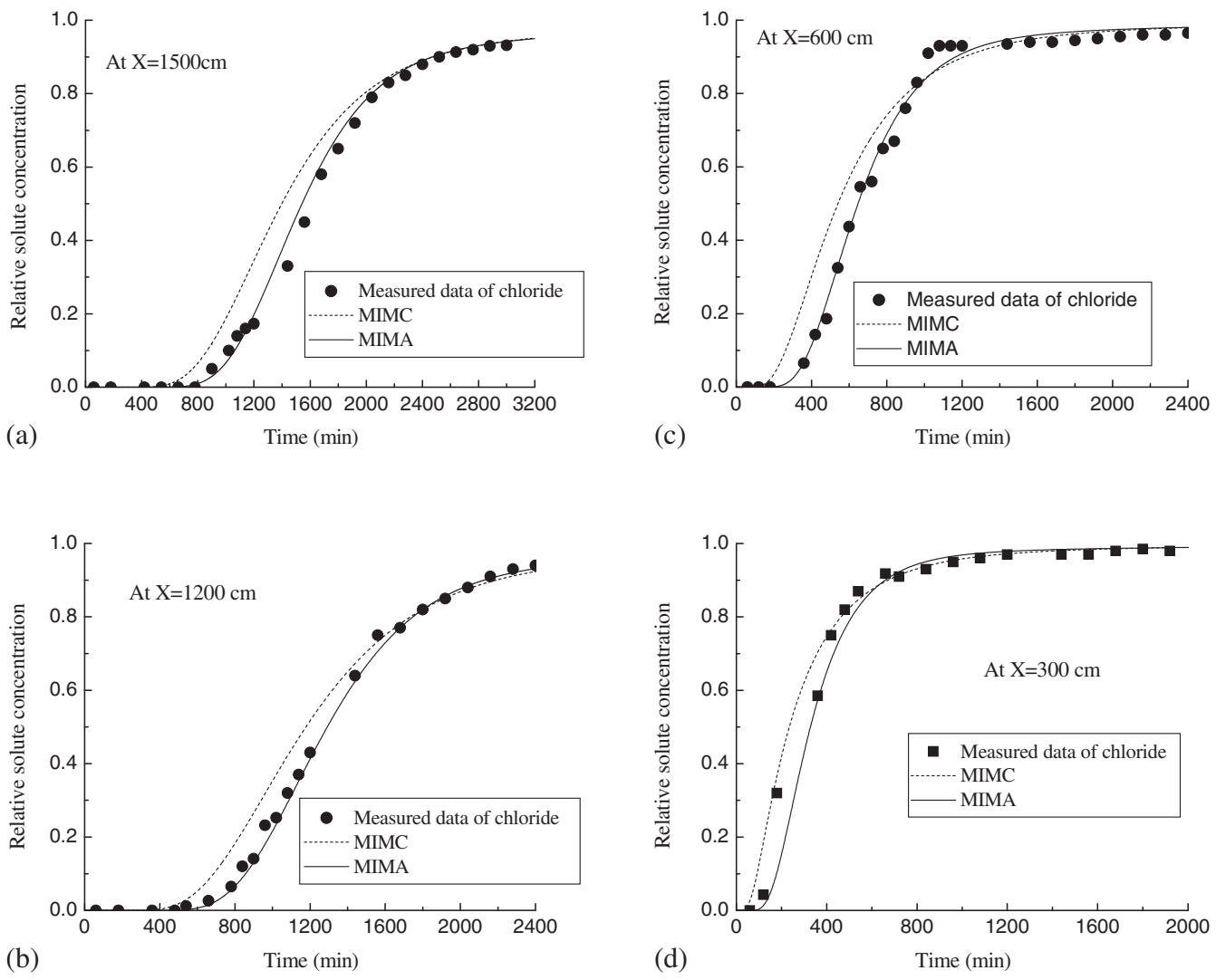

Figure 4. (a) Simulation of observed experimental data of chloride at $1500 \mathrm{~cm}$ down gradient distance using MIMC and MIMA dispersion models. (b) Simulation of observed experimental data of chloride at $1200 \mathrm{~cm}$ down gradient distance using MIMC and MIMA dispersion models. (c) Simulation of observed experimental data of chloride at $600 \mathrm{~cm}$ down gradient distance using MIMC and MIMA dispersion models. (d) Simulation of observed experimental data of chloride at $300 \mathrm{~cm}$ down gradient distance using MIMC and MIMA dispersion models. 
Table 2. Goodness of fit values for Chloride BTC simulation using MIMC and MIMA models.

\begin{tabular}{lcccc}
\hline & \multicolumn{2}{c}{ MIMC } & & \multicolumn{2}{c}{ MIMA } \\
\cline { 2 - 3 } \cline { 5 - 6 } Distance & $r^{2}$ & RMSE & & $r^{2}$ \\
\hline 1500 & 0.9754 & 0.08135 & 0.99647 & 0.02631 \\
1200 & 0.98808 & 0.05685 & 0.998849 & 0.017654 \\
600 & 0.96508 & 0.083148 & 0.99466 & 0.025891 \\
300 & 0.9895 & 0.03891 & 0.9897 & 0.047156 \\
\hline
\end{tabular}

\subsection{Simulation of experimental breakthrough curves of fluoride data}

In order to simulate fluoride experimental data, MPNE transport model is used. For the constant and asymptotic distance-dependent dispersion models, the values of dispersion coefficient and advective region volumetric water content were taken same as estimated from chloride data simulation. However, the value mass transfer coefficient (w) of fluoride is obtained by correcting
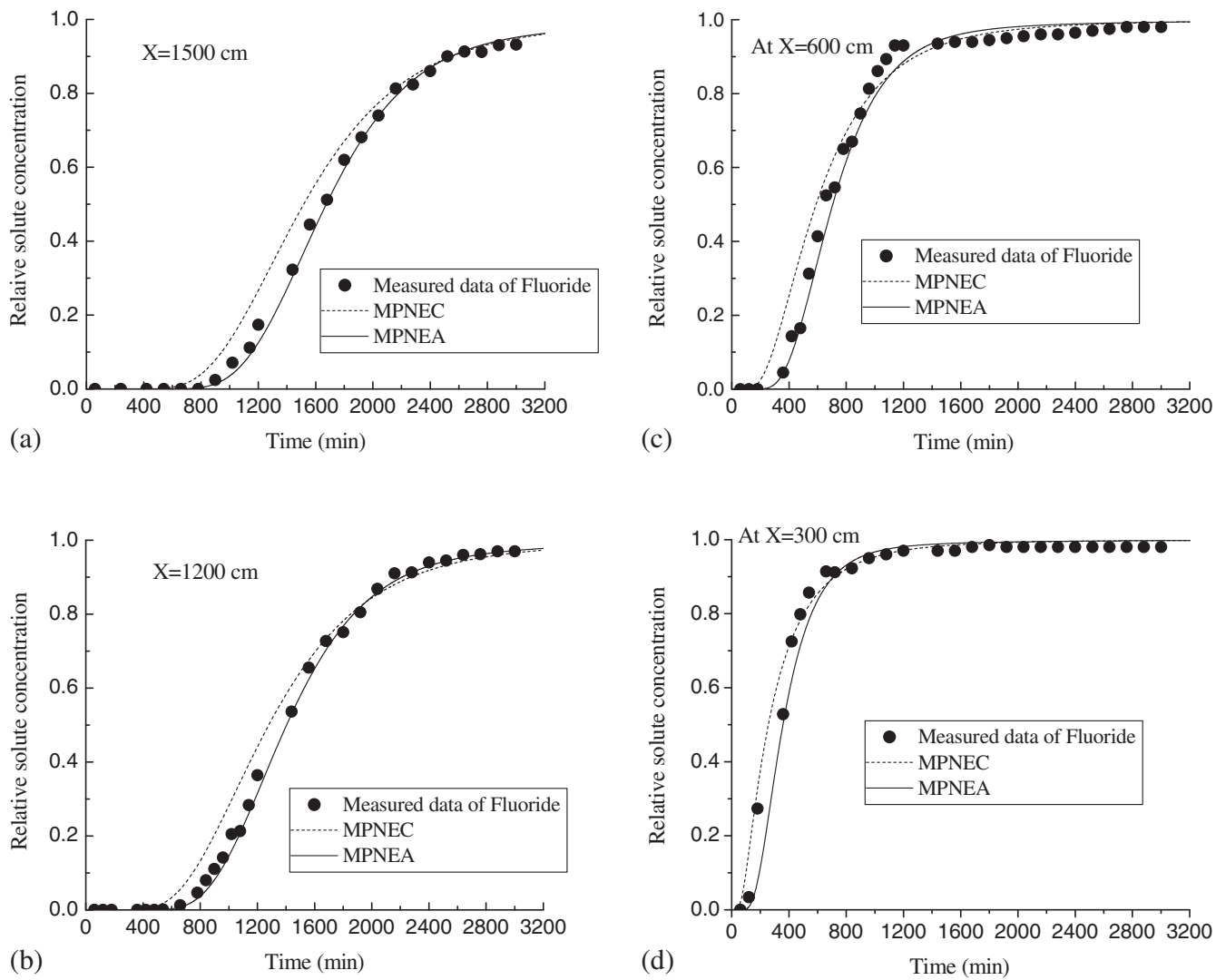

Figure 5. (a) Simulation of observed experimental data of fluoride at $1500 \mathrm{~cm}$ using MPNEC and MPNEA dispersion models. (b) Simulation of observed experimental data of fluoride at $1200 \mathrm{~cm}$ using MPNEC and MPNEA models. (c) Simulation of observed experimental data of fluoride at $600 \mathrm{~cm}$ using MPNEC and MPNEA models. (d) Simulation of observed experimental data of fluoride at $300 \mathrm{~cm}$ using MPNEC and MPNEA models. 
Table 3. Goodness of fit values for Fluoride data simulation using MPNEC and MPNEA.

\begin{tabular}{lccccc}
\hline & \multicolumn{2}{c}{ MPNEC } & & \multicolumn{2}{c}{ MPNEA } \\
\cline { 2 - 3 } \cline { 5 - 6 } Distance & $r^{2}$ & RMSE & & $r^{2}$ & RMSE \\
\hline 1500 & 0.9897 & 0.05438 & & 0.998237 & 0.01865 \\
1200 & 0.98924 & 0.052159 & & 0.99878 & 0.01676 \\
600 & 0.9776 & 0.065424 & & 0.9911 & 0.036972 \\
300 & 0.98617 & 0.039704 & & 0.980404 & 0.0506831 \\
\hline
\end{tabular}

the value of $w=2.31 \mathrm{E}-05$ per min from chloride by multiplying it with ratio of free water diffusion coefficient of fluoride and chloride. Thus the value of mass transfer coefficient for fluoride was obtained as equal to $1.66 \mathrm{E}-05$ per min. Value of $f$ was taken as ratio of advective and non-advective porosity i.e. $f=0.89$. In order to estimate the transport parameters in case of reactive case, it is assumed that $F_{\mathrm{a}}=F_{\mathrm{n}}, K_{\mathrm{a}}=K_{\mathrm{n}}$ and $k_{\mathrm{a} 2}=k_{\mathrm{n} 2}$. The fluoride data at 1500 $\mathrm{cm}$ were first simulated using constant dispersion model. Transport parameters i.e. $F_{\mathrm{a}}, K_{\mathrm{a}}$, and $k_{\mathrm{a} 2}$ were estimated using Levenberg Marquardt nonlinear least square optimization algorithm.

The estimated value of transport parameters is obtained as $F_{\mathrm{a}}=F_{\mathrm{n}}=0.758 ; K_{\mathrm{a}}=K_{\mathrm{n}}=$ $0.021 \mathrm{ml} / \mathrm{g}$; and $k_{\mathrm{a} 2}=k_{\mathrm{n} 2}=0.02$ per min. Using the above estimated parameters Fluoride data were simulated at $1500 \mathrm{~cm}$ using multiprocess non-equilibrium constant dispersion (MPNEC) and asymptotic distance-dependent dispersion (MPNEA) models. Thus all the parameters of Fluoride data were independently simulated. The BTC curves for constant and asymptotic distance dependent models are shown in figure 5a.

It can be seen here that both models estimate BTC very well however, for asymptotic distance dependent models all the parameters were independently estimated. Using the estimated parameters for constant and asymptotic distance-dependent dispersion models, BTC were simulated at $1200 \mathrm{~cm}, 600 \mathrm{~cm}$ and $300 \mathrm{~cm}$ as shown in figure 5b, 5c and 5d respectively. Goodness of fit values for Fluoride simulation using constant and asymptotic distance dependent model is shown in table 3. The computed value of RMSE is smaller in case of MPNEC dispersion model as compared to MPNEA dispersion model. It can be seen that at $1500 \mathrm{~cm}$ and $1200 \mathrm{~cm}$ MPNEC model tends to overestimate the observed BTC. From the simulated results of measured concentration profile, it can be seen that the asymptotic distance-dependent dispersion model fits the experimental data much better as compared to constant dispersion model.

\section{Summary and conclusions}

In this study, semi-analytical solution of multiprocess non-equilibrium transport model with an asymptotic distance-dependent dispersion (MPNEA) is developed for constant concentration type boundary condition. Analytical solution is derived in Laplace domain which has then inverted numerically. To describe the features of multiprocess non-equilibrium transport model, results of experimental breakthrough curves are compared between constant and asymptotic distance-dependent dispersion models. The present semi-analytical solution of MPNEA model was used to describe solute transport in $1500 \mathrm{~cm}$ long heterogeneous soil column. Transport parameters estimated at $1500 \mathrm{~cm}$ was used to simulate breakthrough curves at foregoing distances. Results show that a better fit to the experimental BTC is observed when mass transfer between advective and non-advection region is considered. It was also observed that asymptotic distance-dependent dispersion model gave a good fit to the observed BTC as compared to constant dispersion model. The simulation results demonstrated that multiprocess non-equilibrium 
with constant dispersion model could not adequately describe solute transport in large heterogeneous soil column and it overestimated solute transport dispersion at foregoing distances away from $1500 \mathrm{~cm}$ long soil column. Finally, in this study the development of MPNEA model has been tested by describing solute transport in a laboratory long column experiment. For further research, the present method can be used to analyze other field scale data.

\section{Acknowledgements}

This work was supported by the Department of Science and Technology, New Delhi under research grant number DST-456-CED.

\section{References}

Bear J 1972 Dynamics of fluid in porous media. New York: Elsevier

Berkowitz B, Cortis A, Dentz M and Scher H 2006 Modeling non-Fickian transport in geological formations as a continuous time random walk. Rev. Geophys. 44: RG2003, doi:10.1029/2005RG000178

Brusseau H L, Jessup R E and Rao P S C 1989 Modeling the transport of solutes influenced by multi process non equilibrium. Water Resour. Res. 25: 1971-1988

Brusseau M L, Jessup R E and Rao P S C 1992 Modeling the transport of solutes influenced by multi process nonequilibrium and transformation reactions. Water Resour. Res. 28(1): 175-182

Chen J S, Chen C S, Gau H S and Liu C W 1999 A two-well method to evaluate transverse dispersivity for tracer tests in a radially convergent flow field. J. Hydrol. 223: 175-197

Chen J S, Liu C W and Liao C M 2002 A novel analytical power series solution for solute transport in a radially convergent flow field. J. Hydrol. 266: 120-138

Chen J S, Liu C W, Hsu H T and Liao C M 2003 A Laplace transformed power series solution for solute transport in a convergent flow field with scales dependent Dispersion. Water Resour. Res. 39(8): 1229, doi:10.1029/2003WR002299

Chen J S, Ni C F, Liang C P and Chiang C C 2008 Analytical power series solution for contaminant transport with hyperbolic asymptotic distance-dependent dispersivity. J. Hydrol. 362: 142-149

de Hoog F R, Knight J H and Stokes A N 1982 An improved method for numerical inversion of Laplace transforms. SIAM J. Sci. Stat. Comput. 3(3): 357-366

De Smedt F and Wierenga P J 1979 A generalized solution for solute flow in soils with mobile and immobile water. Water Resour. Res. 15(4): 1137-1141

Dentz M, Kinzelbach H, Attinger S and Kinzelbach W 2000 Temporal behavior of a solute cloud in a heterogeneous porous medium: 2. Spatially extended injection. Water Resour. Res. 36(12): 3605-3614

Furman A and Neuman S P 2003 Laplace-transform analytic element solution of transient flow in porous media. Adv. Water Resour. 26(12): 1229-1237

Gao G, Feng S, Zhan H, Huang G and Ma X 2009a Evaluation of anomalous solute transport in a large heterogeneous soil column with mobile-immobile model. J. Hydrol. Eng. 14(9): 966-974

Gao G, Zhan H, Feng S, Huang G and Mao X 2009b Comparison of alternative models for simulating anomalous solute transport in a large heterogeneous soil column. J. Hydrol. 377(3-4): 391-404

Gao G, Zhan H, Feng S, Fu B, Ma Y and Huang G 2010 A new mobile immobile model for reactive solute transport with scale dependent dispersion. Water Resour. Res. 46: W08533

Gao G, Zhan H, Feng S, Fu B and Huang G 2012 A mobile-immobile model with an asymptotic scaledependent dispersion function. J. Hydrol. 424-425 (2012): 172-183

Gelhar L W 1993 Stochastic subsurface hydrology. Englewood Cliffs, N.J.: Prentice-Hall

Gelhar L W, Welty W and Rehfeldt K R 1992 A critical review of data on field-scale dispersion in aquifers. Water Resour. Res. 28(7): 1955-1974

Goltz M N and Roberts P V 1986 Three-dimensional solutions for solute transport in an infinite medium with mobile and immobile zones. Water Resour. Res. 22(7): 1139-1148 
Guerrero J S P and Skaggs T H 2010 Analytical solution for one-dimensional advection-dispersion transport equation with distance-dependent coefficients. J. Hydrol. 390: 57-65

Huang K, Toride N and van Genuchten M Th 1995 Experimental investigation of solute transport in large, homogeneous and heterogeneous saturated soil column. Transp. Porous Media 18(2): 283-302

Huang H, Huang Q and Zhan H 2006 Evidence of one dimensional scale-dependent fractional advectiondispersion. J. Cont. Hydrol. 85(1-2): 53-71

Huang K, van Genuchten M T and Zhang R 1996 Exact solutions for One dimensional transport with asymptotic scale dependent dispersion. Appl. Math. Model. 20: 298-308

Khan A U and Jury W A 1990 A laboratory study of the dispersion scale effect in column outflow experiments. J. Contam. Hydrol. 5: 119-131

Kreyszig E 1999 Advanced engineering mathematics. Eighth ed. New York: John Wiley

Logan J D 1996 Solute transport in porous media with scale-dependent dispersion and periodic boundary conditions. J. Hydrol. 184(3-4): 261-276

Lapidus L and Amundson N R 1952 Mathematics of adsorption in beds. VI. The effect of longitudinal diffusion in ion exchange and chromatographic columns. J Phys. Chem. 56: 984-988

Mishra S and Parker J C 1990 Analysis of solute transport with a hyperbolic scale dependent dispersion model. Hydrol. Process. 4: 45-57

Moench M 1991 Social issues in Western U.S. groundwater management: An overview. Oakland: Pacific Institute

Molz F J, Guven O and Melville J G 1983 An examination of scale-dependent dispersion coefficients. Ground Water 21(6): 715-725

Park E and Zhan H 2003 Hydraulics of horizontal wells in fractured shallow aquifer systems. J. Hydrol. 281: $147-158$

Pickens J F and Grisak G E 1981a Scale-dependent dispersion in a stratified granular aquifer. Water Resour Res 17(3): 1191-1211

Pickens J F and Grisak G E 1981b Modeling of scale-dependent dispersion in hydrogeologic systems. Water Resour Res 17(6): 1701-1711

Schulze-Makuch D 2005 Longitudinal dispersivity data and implications for scaling behavior. Ground Water 43(2): 443-456

Vanderborght J and Vereecken H 2007 Review of dispersivities for transport modelling in soil. J. Vadose Zone 6: 29-52

Valocchi A J 1985 Validity of the local equilibrium assumption for modeling sorbing solute transport through homogeneous soils. Water Resour. Res. 21(6): 808-820

van Genuchten M Th and Wierenga P J 1976 Mass transfer studies in sorbing porous media: I. Analytical solutions. Soil Sci. Soc. Am. J. 40(3): 473-480

van Genuchten M Th and Wierenga P J 1977 Mass transfer studies in sorbing porous media: Experimental evaluation with tritium. Soil. Sci. Soc., Am. J. 41(2): 272-278

van Genuchten M Th and Alves W J 1982 Analytical solutions of the one-dimensional convective-dispersive solute transport equation. US Dept. Agriculture Tech. Bull. No. 1661 151p

Yates S R 1990 An analytical solution for one-dimensional transport in heterogeneous porous media. Water Resour. Res. 26(10): 2331-2338

Yates S R 1992 An analytical solution for one-dimensional transport in heterogeneous porous media with an exponential dispersion function. Water Resour. Res. 28(8): 2149-2154 